\title{
Pasireotide LAR and Octreotide LAR Maintain Inhibition of GH and IGF-1 in Patients With Acromegaly: 12-Month Extension Phase of a Randomized, Double-Blind, Multicenter, Phase III Study
}

M Sheppard, ${ }^{1}$ M Bronstein, ${ }^{2}$ P Freda, ${ }^{3}$ O Serri, ${ }^{4}$ L De Marinis, ${ }^{5}$ L Naves, ${ }^{6}$ L Rozhinskaya, ${ }^{7}$ K Hermosillo Resendiz, ${ }^{8}$ M Ruffin, ${ }^{9}$ K Asubonteng, ${ }^{8}$ A Colao ${ }^{10}$ 'Centre for Endocrinology, Diabetes and Metabolism, University of Birmingham, Edgbaston, Birmingham, UK; ${ }^{2}$ Neuroendocrine Unit, Division of Endocrinology and Metabolism, University of São Paulo Medical School, São Paulo, Brazil; 'Department of Medicine, Columbia University College of Physicians \& Surgeons, New York, New York, USA; ‘Service of Endocrinology, Centre Hospitalier de l'Université de Montreal, Notre-Dame Hospital, University of Montreal, Montreal, Canada; 'Section of Endocrinology, Department of Internal Medicine, Università Cattolica del Sacro Cuore, Rome, Italy; ${ }^{6}$ Department of Endocrinology, Internal Medicine, University of Brasilia,
Brasilia, Brazil; 'Department of Neuroendocrinology and Bone Diseases, Endocrinology Research Centre, Moscow, Russia; ${ }^{\circ}$ Clinical Development, Novartis Pharmaceuticals Corporation, Florham Park, New Jersey, USA; ${ }^{\circ}$ Clinical Development, Oncology Business Unit, Novartis Pharma AG, Basel, Switzerland; ${ }^{10 D i p a r t i m e n t o ~ d i ~ M e d i c i n a ~ C l i n i c a ~ e ~ C h i r u r g i a, ~ U n i v e r s i t a ̀ ~ F e d e r i c o ~ I I ~ d i ~ N a p o l i, ~ N a p l e s, ~ I t a l y ~}$

\section{INTRODUCTION}

Acromegaly, if left untreated, is associated with a 2 to 2.5 times Acreased mortality risk compared with the general population. Reducing growth hormone $(\mathrm{GH})$ levels $<2.5 \mu \mathrm{g} / \mathrm{L}$ and insulin-like $\mathrm{g}$

Pasireotide, a multireceptor-targeted somatostatin analogue (SSA) that has broader somatostatin receptor binding profile (high affinity binding to 4 out of 5 somatostatin receptor subtypes) than the currently available SSA, provides effective control of GH and IGF-1 levels in patients with $\mathrm{GH}$-secreting pituitary adenomas

Pasireotide long-acting release (LAR) demonstrated significantly superior biochemical control $(P=0.007)$ than octreotide LAR in a randomized, double-blind, 12-month trial in 358 medically naïve patients with acromegaly.

An extension phase to this study allowed patients to continue receiving their randomized therapy or crossover at month 13 depending on their GH and IGF-1 levels as well as whether they were receiving clinical benefit from the study drug.

This poster reports the results up to month 26 in patients with clinica benefit or GH $<2.5 \mu \mathrm{g} / \mathrm{L}$ and IGF-1 supper limit of normal (ULN) at month 13 who continued receiving their randomized therapy in

extension phase.

Poster 847 reports the results in patients who switched treatments at month 13 .

\section{METHODS}

Study Design

- Medically naïve patients (either post-pituitary surgery or de novo with visible pituitary adenoma on magnetic resonance imaging who refused pituitary surgery or for whom plutiny surgery was contraindicated) with active acromegaly (GH $>5 \mu \mathrm{g} / \mathrm{L}$ or GH nadir $\geq 1 \mu \mathrm{g} / \mathrm{L}$ post-oral glucose tolerance test, and $G_{F}-1>U L N$ ) were eligible for enrollment into the 12-month core study.

Patients were randomized to pasireotide LAR $40 \mathrm{mg} / 28$ days or octreotide LAR $20 \mathrm{mg} / 28$ days. Dose titration to pasireotide LAR $60 \mathrm{mg} / 28$ days or octreotide LAR $30 \mathrm{mg} / 28$ days permitted, but not mandatory, at month 3 or 7 . Dose decreases were permitted for tolerability issues (Figure 1 ).

Figure 1. Study Design

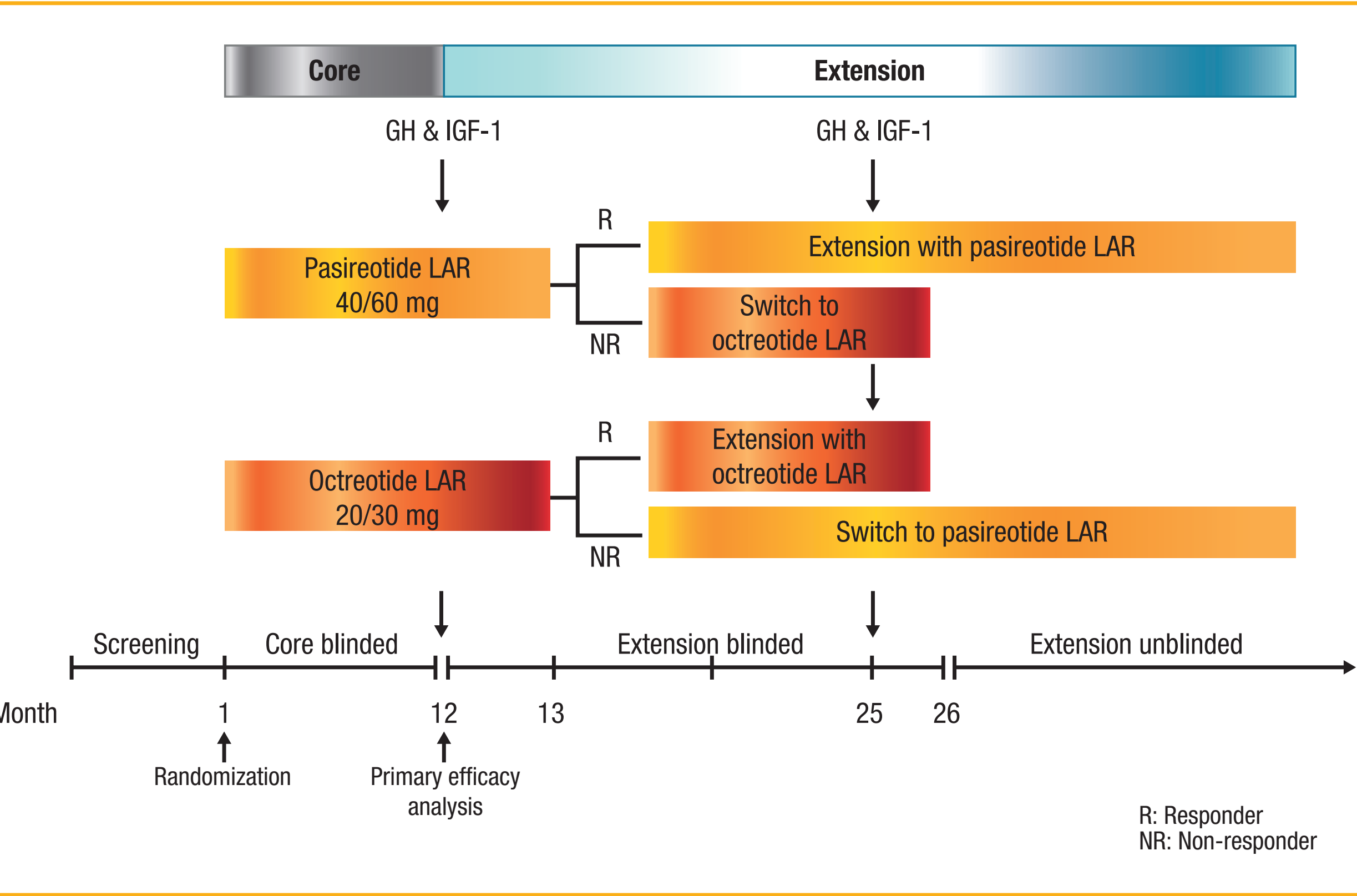

A protocol amendment implemented shortly after the trial had begun, established a double-blind extension phase whereby patients could either remain on their randomized therapy or crossover to the opposite treatment.

Prior to the protocol amendment, patients who were inadequately controlled with octreotide LAR could switch to pasireotide LAR at month 12 , but not vice versa.

Following this amendment, patients with GH $<2.5 \mu \mathrm{g} / \mathrm{L}$ and IGF-1 $\leq U L N$ at month 12 could continue on their randomized therapy as could patients considered by the investigator to be achieving clinical benefit.

Patients entering the extension were followed up to month 26 (core plus extension).

Last assessment of GH, IGF-1, and tumor volume was performed at month 25

Last assessment of safety and monthly acromegaly symptom scores was performed at month 26

Dose escalation to pasireotide LAR $60 \mathrm{mg}$ or octreotide LAR $30 \mathrm{mg}$ was permitted, but not mandatory, at any time during extension if GH $\geq 2.5 \mu \mathrm{g} / \mathrm{L}$ and/or IGF-1 >ULN. Dose decreases were permitted for tolerability issues.

Study Objectives and Endpoints

The primary objective of the core study was to demonstrate the superiority of pasireotide LAR over octreotide LAR in providing $\mathrm{GH}<2.5 \mu \mathrm{g} / \mathrm{L}$ and normal IGF-1 at month 12.

The objective of the extension study was to assess the effect of pasireotide LAR and octreotide LAR as long term treatment on the following efficacy endpoints.

Proportion of patients with a reduction of mean GH level to $<2.5 \mu \mathrm{g} / \mathrm{L}$ and normalization of IGF-1 (age and sex related) at months 16 and 25 Proportion of patients achieving $\mathrm{GH}<2.5 \mu \mathrm{g} / \mathrm{L}$ at months 16 and 25 Proportion of patients achieving normal IGF-1 at months 16 and 25 Change from core baseline in (i) GH levels (ii) IGF-1 levels,

(iii) tumor volume, and (iv) severity scores (0 to 4 scale) of acromegaly symptoms (headache, fatigue, perspiration, paresthesia, and osteoarthralgia), over time

Safety and tolerability of pasireotide LAR and octreotide LAR as long term treatment was evaluated. Safety assessments included monitoring of adverse events (AEs), as well as hematology, blood chemistry and urinalysis parameters.

\section{RESULTS}

Patients

Of the 358 patients who entered the core phase, 120 patients entered the extension in their randomized arm and continued receiving randomized treatment (Figure 2).

Figure 2. Patient Disposition

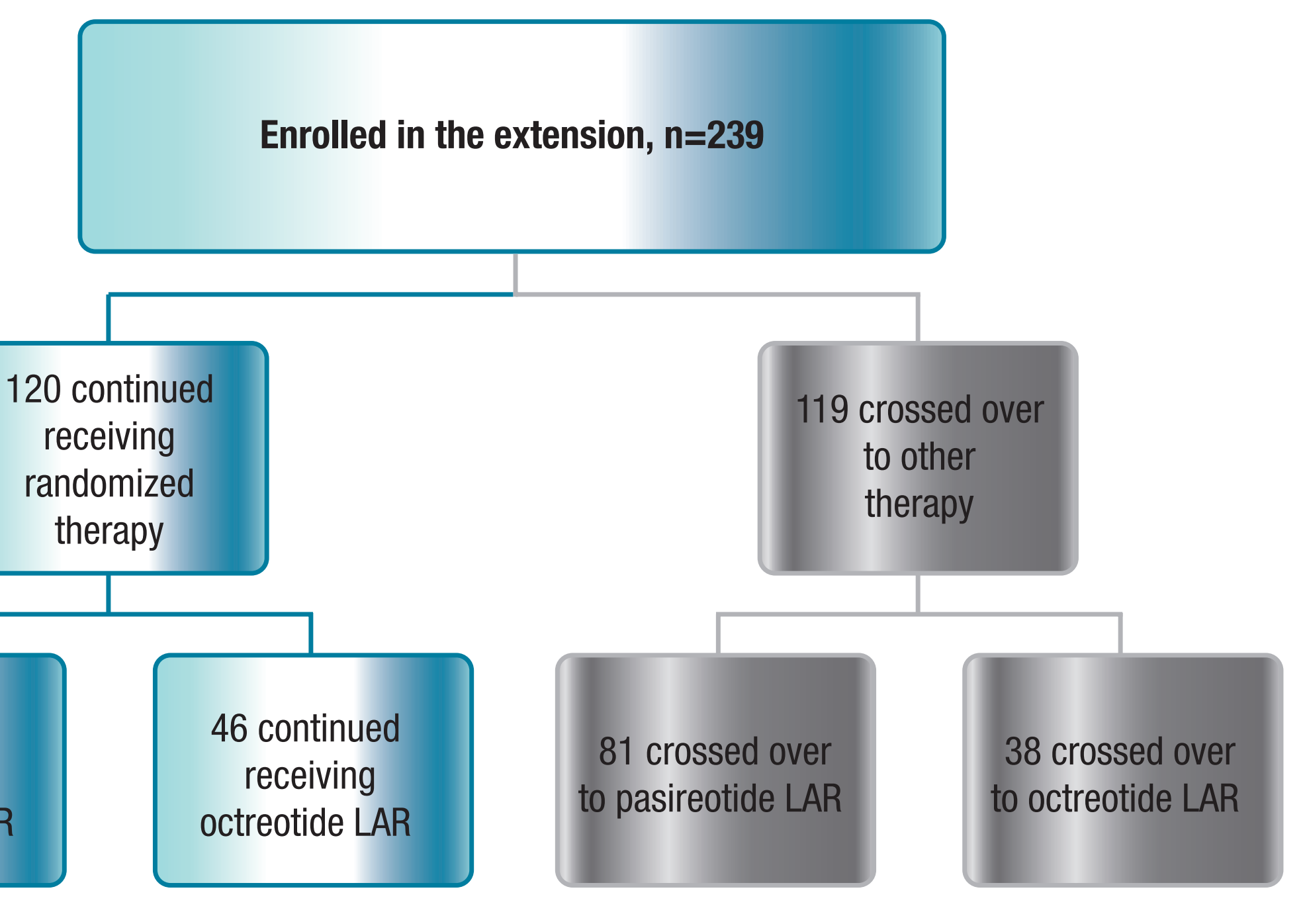

Efficacy

Mean duration of exposure was 527 days in the pasireotide LAR arm and 415 days in the octreotide LAR arm.

Suppression of GH and IGF-1 was maintained throughout the extension in both the treatment arms (Figure 3).

Figure 3. Median (A) GH and (B) IGF-1 Levels During Treatment

(A) - Pasireotide LAR
- Octreotide LAR

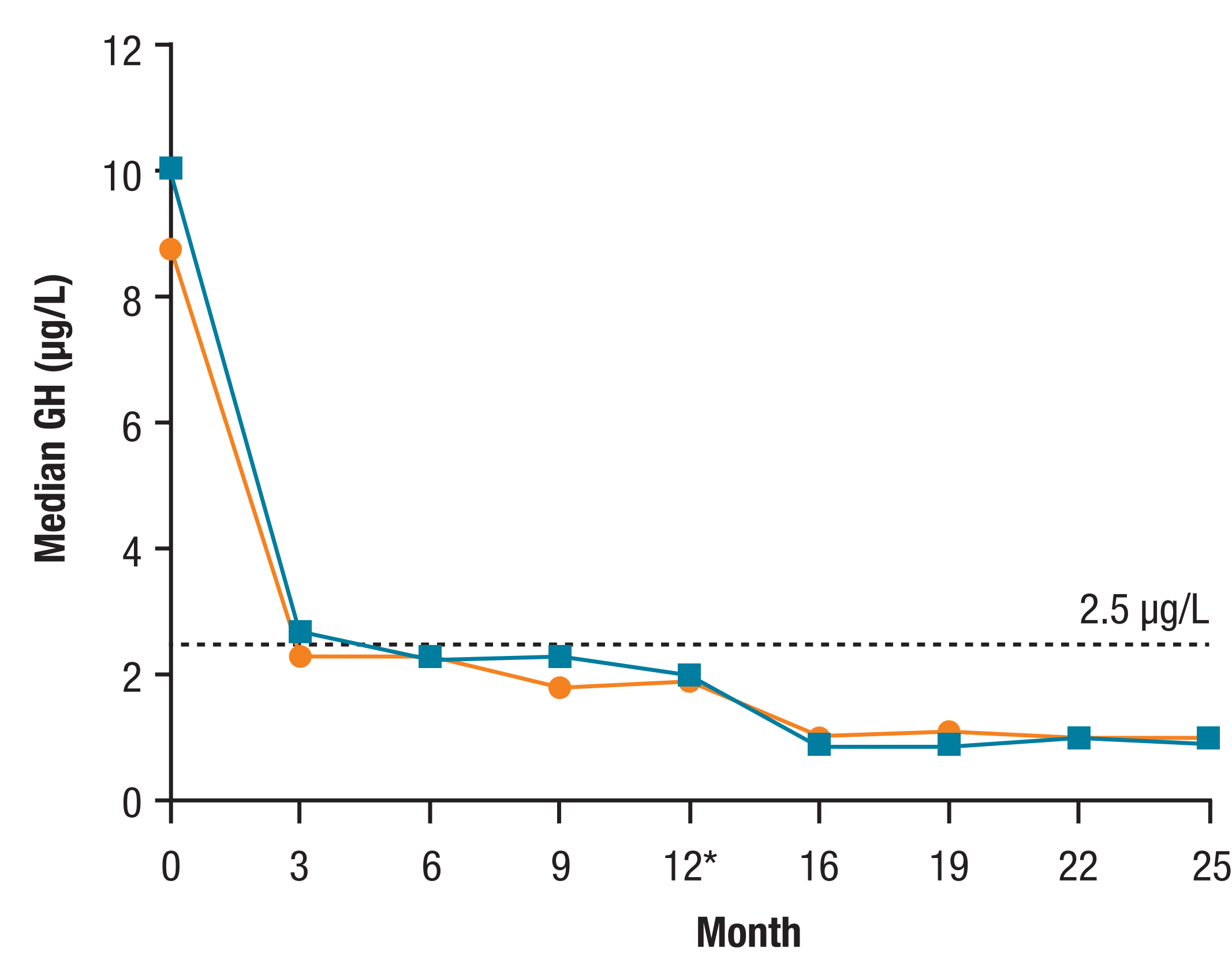

$\begin{array}{lllllllll}\text { Pasireotide LAR, n } 167 & 164 & 151 & 136 & 136 & 64 & 62 & 60 & 62\end{array}$

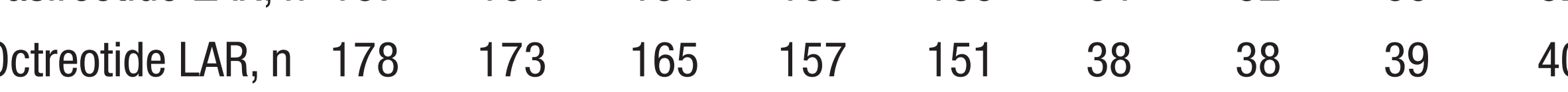
(B)

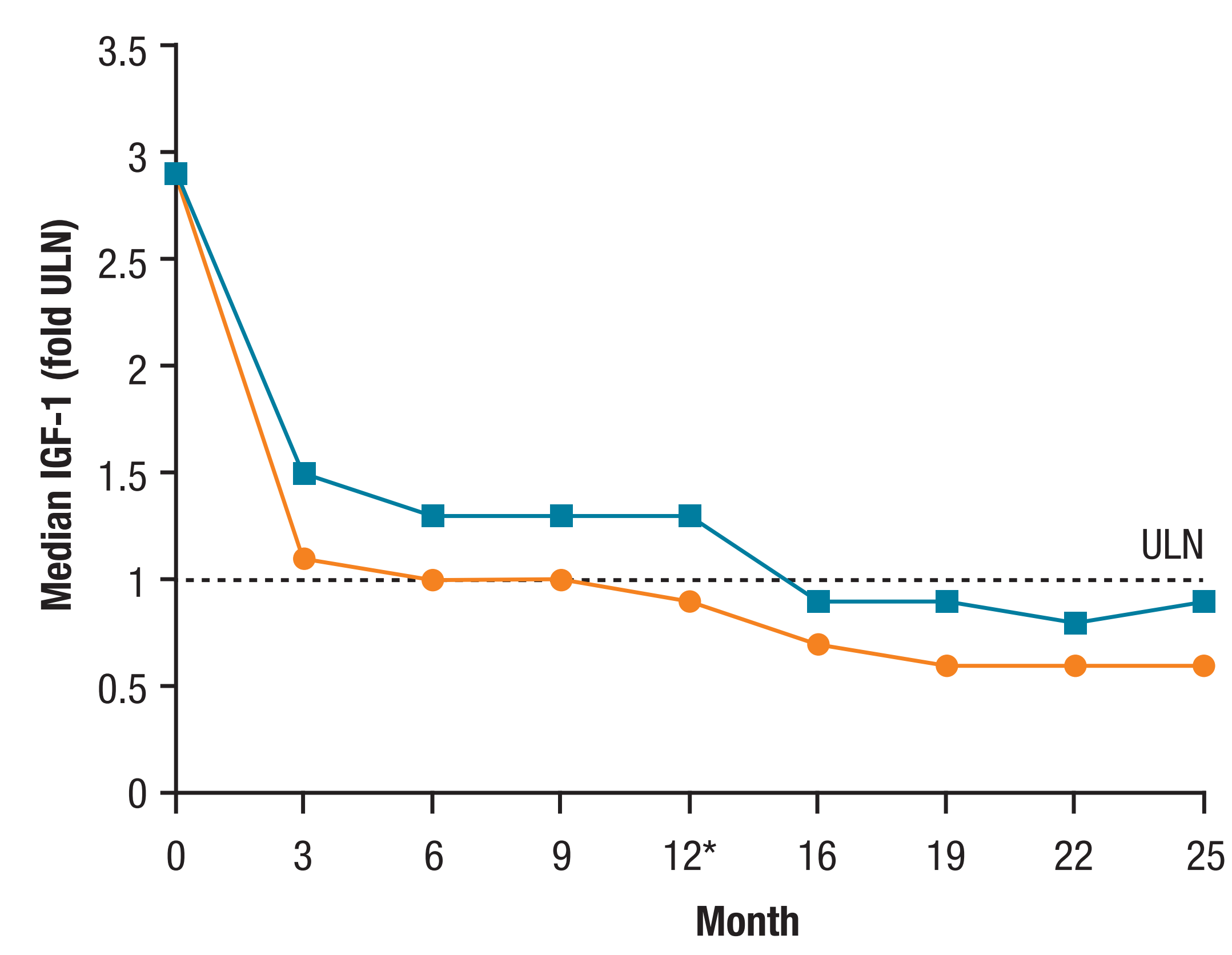

$\begin{array}{lllllllll}\text { Pasireotide LAR, n } 176 & 164 & 150 & 138 & 136 & 66 & 63 & 60 & 62\end{array}$ $\begin{array}{llllllllll}\text { Octreotide LAR, } \mathrm{n} & 182 & 174 & 169 & 159 & 151 & 38 & 38 & 39 & 40\end{array}$

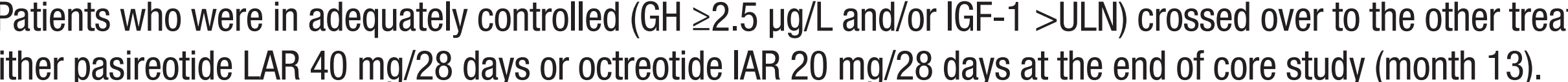

Median percentage change in GH from core baseline to month 25 was $-83 \%$ in patients in the pasireotide LAR and $-86 \%$ in patients in the octreotide LAR treatment arm.

Median percentage change in IGF- 1 from core baseline to month 25 was $-71 \%$ with pasireotide LAR and $-64 \%$ with octreotide LAR.

At month $25,48.6 \%$ of pasireotide LAR and $45.7 \%$ of octreotide LAR patients achieved $\mathrm{GH}<2.5 \mu \mathrm{g} / \mathrm{L}$ and normal IGF-1 (Table 1).

At month $25,60.8 \%$ of pasireotide LAR patients and $52.2 \%$ of octreotide LAR patients had mean GH levels $<2.5 \mu \mathrm{g} / \mathrm{L}$ and IGF- $1 \leq \mathrm{ULN}$.

Tumor volume decreased from core baseline to month 25 by a mean (SD) of $-51.8 \%(20.8 \%)$ in the pasireotide LAR arm and $-55.0 \%(21.3 \%)$ in the octreotide LAR arm.

A significant $(\geq 20 \%)$ tumor volume reduction from extension baseline to month 25 was seen in $74.7 \%$ of pasireotide LAR and $71.6 \%$ of octreotide AR patients.

Both the treatments improved severity scores of acromegaly symptoms.
Table 1. Biochemical Response Rates in Patients who Continued Receiving Their Randomized Dose up to Month 26

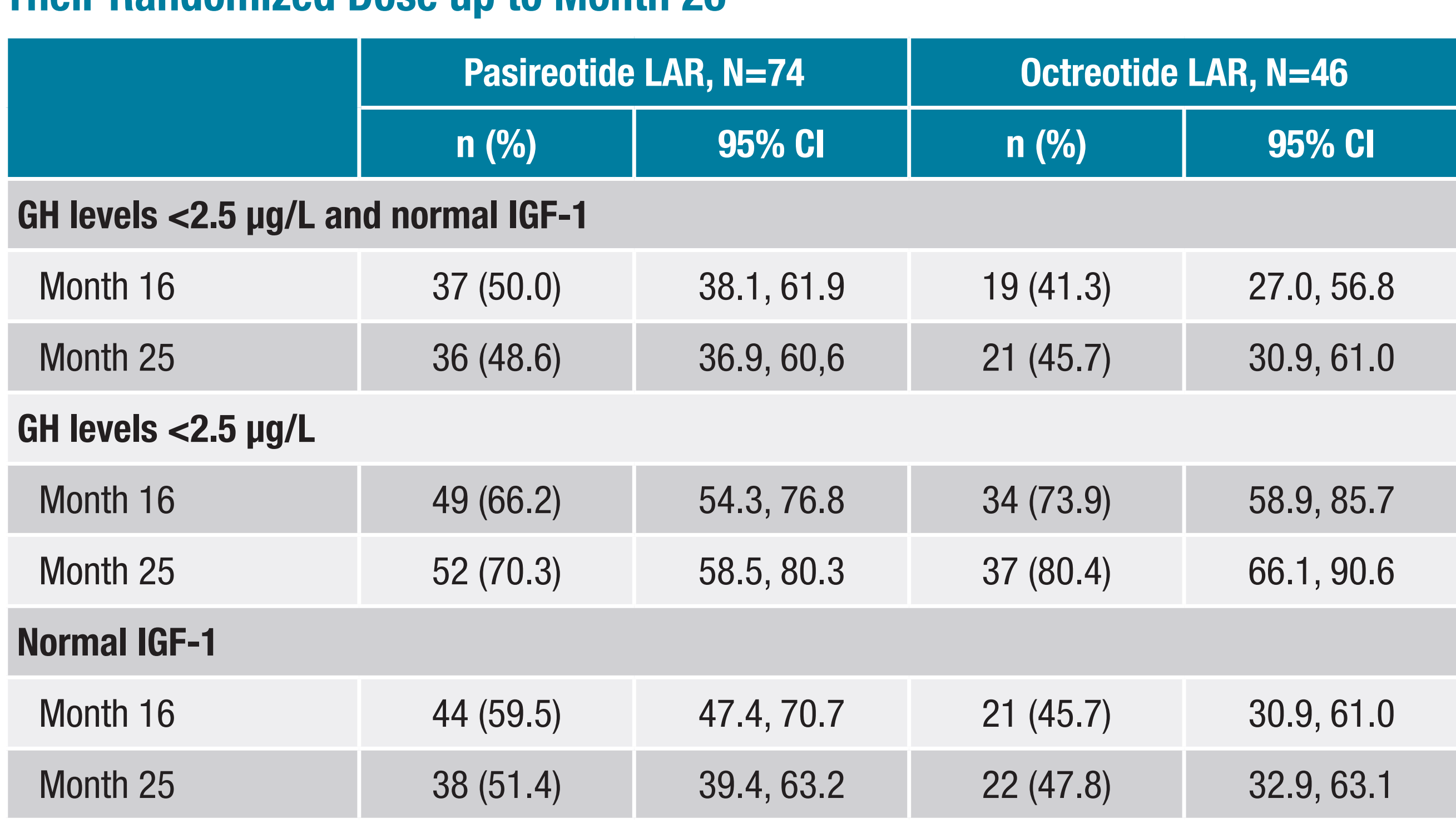

Safety

Overall, 23/74 (31.1\%) and $10 / 46$ (21.7\%) patients in the pasireotide LAR and octreotide LAR arms who continued receiving their randomized months 12 and 26 .

Most common reas

Two deaths were reported during the extension phase (major depression in a pasireotide LAR patient, sepsis in an octreotide LAR patient). Both were considered to be unrelated to study drug by the investigator (Table 2).

Table 2. Most Commonly Reported Adverse Events (>15\% in Total in Either Treatment Arm) From Core Baseline to Month 26

\begin{tabular}{|c|c|c|c|c|}
\hline & \multicolumn{2}{|c|}{ Pasireotide LAR $\left(\mathrm{N}=178^{*}\right)$} & \multicolumn{2}{|c|}{ Octreotide LAR $\left(\mathrm{N}=180^{*}\right)$} \\
\hline & $\begin{array}{c}\text { All grades } \\
\text { n (\%) }\end{array}$ & $\begin{array}{c}\text { Grade } 3 / 4 \\
\text { n (\%) }\end{array}$ & $\begin{array}{c}\text { All grades } \\
\text { n ( } \%)\end{array}$ & $\begin{array}{c}\text { Grade } 3 / 4 \\
n(\%)\end{array}$ \\
\hline Diarrhea & $71(39.9)$ & $1(0.6)$ & $81(45.0)$ & $5(2.8)$ \\
\hline Cholelithiasis & $58(32.6)$ & $3(1.7)$ & $71(39.4)$ & $3(1.7)$ \\
\hline Headache & $41(23.0)$ & $2(1.1)$ & $49(27.2)$ & $5(2.8)$ \\
\hline Abdominal pain & $33(18.5)$ & $1(0.6)$ & $44(24.4)$ & 0 \\
\hline Hyperglycemia & $55(30.9)$ & $6(3.4)$ & $18(10.0)$ & $1(0.6)$ \\
\hline Alopecia & $34(19.1)$ & 0 & $36(20.0)$ & 0 \\
\hline Nasopharyngitis & $32(18.0)$ & 0 & $29(16.1)$ & 0 \\
\hline Nausea & $27(15.2)$ & $1(0.6)$ & $41(22.8)$ & 0 \\
\hline Diabetes mellitus & $39(21.9)$ & $9(5.1)$ & 8 (4.4) & 0 \\
\hline
\end{tabular}

Mean glucose and glycosylated hemoglobin $\left(\mathrm{HbA}_{1 \mathrm{C}}\right)$ increased in the (1) stable to 26 months. In octreotide LAR arm, a smaller and more gradual increase in mean glucose and $\mathrm{HbA}$ was observed, which peaked at months 9 to 12 and remained relatively stable up to month 26 .

Hyperglycemia-related AEs (including but not limited to hyperglycemia, hypoglycemia, diabetes mellitus, and increased $\mathrm{HbA}_{1 \mathrm{C}}$ ) was the only

Proportion of patients with grade 3 or 4 hyperglycemia-related AEs was higher with pasireotide LAR than octreotide LAR (16/178 [9.0\%] vs 3/180 [1.7\%]), and similar to that reported in the core phase.

\section{CONCLUSIONS}

Pasireotide LAR was significantly superior to octreotide LAR in providing biochemical control in the 12-month core phase of the study. During the extension phase, suppression of GH and IGF-1 levels was maintained in both the treatment arms.

No new safety findings were reported during the extension phase compared with the core study.

The safety profile of pasireotide LAR and octreotide LAR was similar, except for the degree of hyperglycemia.

These results suggest that pasireotide LAR and octreotide LAR provide long-term inhibition of GH and IGF-1 in patients with acromegaly.

\section{REFERENCES}

Katznelson Let al. Endocr Pract 2011;17:636-646,
Bruns C et al. Eur J Endocrinol 2002;146:707-716.

Melmed Set al. Pituitary 2010;13:18-28.

Pyronnet Set al. Mol Cell Endocrinol 2008;286:230-237.
Colao A et al. Endocrine Abstracts 2012;29:0C1.1

\section{ACKNOWLEDGEMENTS}

We thank Andrew Jones, Mudskipper Business Ltd. (funded by Novartis Pharmaceuticals Corporation) and Madhoolika Nema, Novartis Healthcare Pvt. Ltd. for providing medical edit
assistance and Hareesh Cheela, Novartis Healthcare Pvt. Ltd. for graphical/poster layout

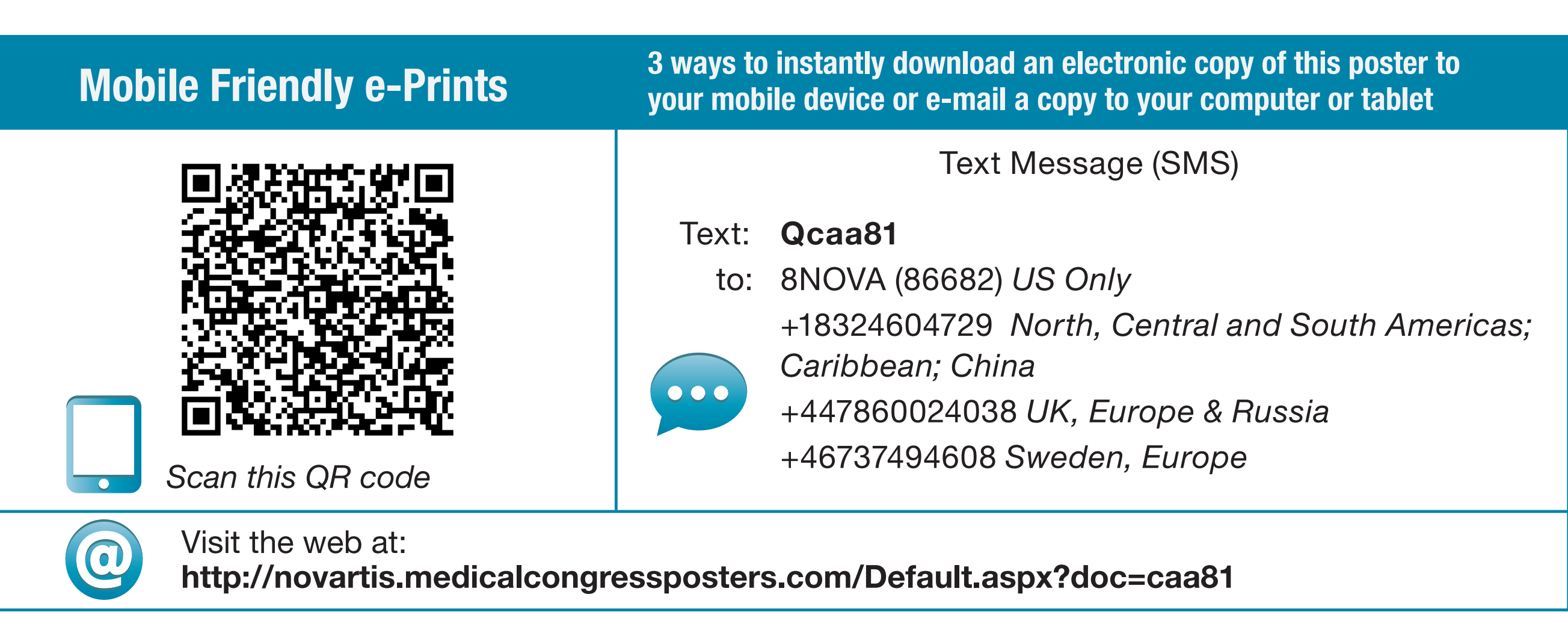

